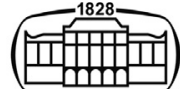

AKADÉMIAI KIADÓ

\title{
Percutaneous aspiration-injection-reaspiration (PAIR) for the treatment of abdominal cysts: Initial report in sheep and goat
}

\section{Acta Veterinaria Hungarica}

$68(2020) 4,393-398$

DOI:

$10.1556 / 004.2020 .00059$

(c) 2021 Akadémiai Kiadó, Budapest

\section{ORIGINAL RESEARCH} PAPER

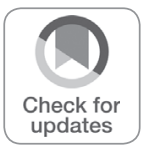

*Corresponding author. Tel.: +91941 9095830.

E-mail: fazili_mr@yahoo.co.in

\author{
NIDA HANDOO ${ }^{1}$, MUJEEB UR REHMAN FAZILI $^{2 *} \odot$, \\ MOHAMMAD ABRAR GAYAS ${ }^{1}$, HAKIM ATHAR ${ }^{1}$ and \\ RAJA TARIQ ${ }^{3}$
}

\footnotetext{
${ }^{1}$ Division of Veterinary Surgery and Radiology, Faculty of Veterinary Sciences and Animal Husbandry, SKUAST, Kashmir, Srinagar, Jammu and Kashmir, India

${ }^{2}$ Division of Veterinary Clinical Complex, Faculty of Veterinary Sciences and Animal Husbandry, SKUAST, Kashmir, H. No. 78, HIG Colony, Bemina, Srinagar, 190018 India

${ }^{3}$ Division of Animal Genetics and Breeding, Faculty of Veterinary Sciences and Animal Husbandry, SKUAST, Kashmir, Srinagar, Jammu and Kashmir, India
}

Received: March 27, 2020 • Accepted: October 14, 2020

Published online: January 16, 2021
KEYWORDS

PAIR technique, sheep, goat, hypertonic saline, sclerotherapy, ultrasonography

\section{INTRODUCTION}

With the introduction of imaging techniques in food animal practice, it has now become possible to diagnose several abdominal focal lesions including cysts (Hussein and Elrashidy, 2014). Abdominal cysts in sheep are mainly of parasitic origin. Taenia hydatigena and Echinococcus granulosus are tapeworms of dogs. The metacestode (larval) stages of these parasites and Cysticercus tenuicollis hydatid cysts develop frequently in the intermediate host (sheep and goats). Sheep infested with cystic echinococcosis are the most important reservoir of human hydatidosis. In human medicine, the current treatment options for abdominal cysts include surgery, laparoscopy and sclerotherapy by percutaneous aspiration-injection-reaspiration (PAIR) techniques. As compared to conventional surgery, minimally invasive 
procedures are more popular currently (Cai et al., 2016). Although laparoscopy is as effective as open surgery, but its requirement for expensive equipment, surgeon expertise and general anaesthesia of the patient preclude its use (Yoder and Wolf, 2004). As compared to laparoscopy, the PAIR procedure is minimally invasive, inexpensive, reduces surgical time and hospital stay, and leads to lower morbidity and mortality (Khuroo et al., 1991, 1997; Ustunsoz et al., 1999; Aygun et al., 2001; Boland et al., 2003; Nasseri et al., 2006; Cai et al., 2016). The most frequently used sclerotic agents include $15-30 \%$ hypertonic saline and 95\% ethanol (Golemanov et al., 2011). Hypertonic saline exerts a scolicidal effect by creating a strong osmotic gradient across the outer cuticular membrane of the protoscolex in the parasitic cysts, leading to its lysis.

Ultrasonography (USG), the most dependable diagnostic tool for abdominal cysts, is now available in most of the rural field veterinary institutions in the developing countries. Interventional USG could therefore be adopted for the benefit of the poor farmers and their livestock. As per the available literature, the use of the PAIR technique has not been reported in food animal clinical practice to date. Only one experimental trial, with an objective to develop a model for human disease management, involved sheep (Akhan et al., 1993). In that study, the animals were subjected to ultrasound-guided injection of hypertonic saline, followed by catheterisation and sclerotherapy of the cysts using alcohol. Unlike in the past, many small ruminants are now kept as pets in urban areas of the developing countries. Their owners are interested in the use of minimally invasive modern disease diagnostic and treatment protocols. A study was therefore planned to evaluate the feasibility of the PAIR procedure using 20\% hypertonic saline solution as sclerotic agent in the management of abdominal cysts in sheep and goats.

\section{MATERIALS AND METHODS}

Five adult ewes and a doe, aged 3-4 years, found to have abdominal cysts (one cyst/animal) on survey ultrasonography using linear and sector transducers (5-10 MHz) of a real-time B-mode ultrasound machine (Esaote Mylab 40 Vet) were included in the study. For confirmation, USG was repeated twice with an interval of one week. All the animals belonged to the Mountain Research Centre on Sheep \& Goats (MRCSG), SKUAST-Kashmir. They were maintained under standard management conditions in an organised university farm. In this temperate zone, sheep spend their days (from March to November) in the open field grazing in Himalayan foothills around the villages. During the harsh snowy winter months (from December to February) they are kept indoors and stall fed using hay and concentrate. In addition to the consent of the In-charge Professor of the MRCSG, permission to undertake the protocol was obtained from the Institutional Animal Ethics Committee (IAEC).

The animals were fasted overnight prior to the third USG examination. Thorough clinical examination was followed by the collection of blood $(5.0 \mathrm{~mL})$ by jugular venipuncture in two vials; one with anticoagulant for routine haematological parameters and the second for the separation of serum to be used later for biochemical analysis. A wide abdominal area was again shaved and scrubbed. The animals were restrained physically in standing position, and a copious volume of USG gel was applied in the shaved area. USG examination was performed to relocate the cysts and record their diameter and volume. The gel was then wiped off and an antiseptic (Isopropyl Alcohol, 90\%, Doctor's Spirit, Neeleshawar Surgical, India) was applied locally. The site in the abdominal wall nearest to the cyst was injected with $1.0 \mathrm{~mL}$ of $2 \%$ lignocaine hydrochloride (Lox, Neon Laboratories Limited, India) and rescrubbed properly. The USG probe was then placed in the immediate next intercostal space after applying a copious volume of gel. It was kept static after focusing at the nearest border of the cyst. A sterile hypodermic needle (G-18) attached to a $10.0-\mathrm{mL}$ sterile Dispovan syringe was passed via the anaesthetised site (perpendicular to the transducer plan) till it crossed the abdominal wall (Fig. 1). The needle was pushed slowly and its tip carefully guided to enter the cyst (Figs. 2 and 3). Approximately $50 \%$ of the cyst fluid was aspirated. Keeping the needle undisturbed, the syringe was disengaged and replaced with another one loaded with $20 \%$ hypertonic saline. Depending on the size of the cyst, 1.0-5.5 mL fluid was aspirated and $0.5-2.0 \mathrm{~mL}$ of $20 \%$ hypertonic saline solution infused. The needle with the attached Dispovan syringe was kept undisturbed for $10 \mathrm{~min}$. The maximum possible volume of the cystic content was subsequently reaspirated and the hypodermic needle withdrawn. The animals were injected meloxicam $(0.5 \mathrm{mg} / \mathrm{kg}$, Melonex, Intas Animal Health, India) and amoxicillin-dicloxacillin $(10.0 \mathrm{mg} / \mathrm{kg}$, Moxel-D, Alembic, India), both intramuscularly (IM).

All the animals were re-evaluated by USG 7 days later. The animals showing no reduction in the cyst size were subjected to the second procedure following the protocol adopted the first time. USG evaluation was repeated on day 30 and day 90 following the first procedure in all the animals.

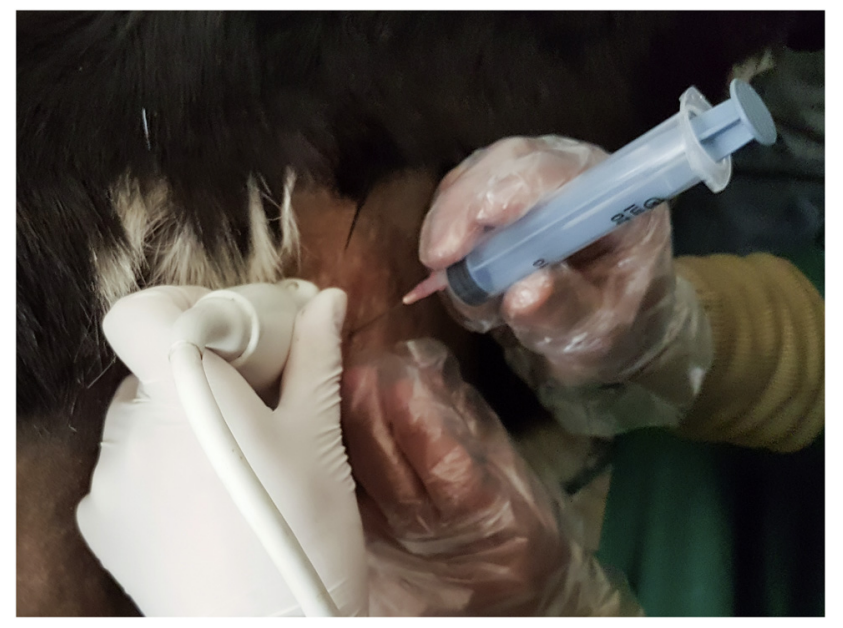

Fig. 1. Ultrasound-guided percutaneous aspiration-injection-reaspiration (PAIR) procedure in a goat with an abdominal cyst 


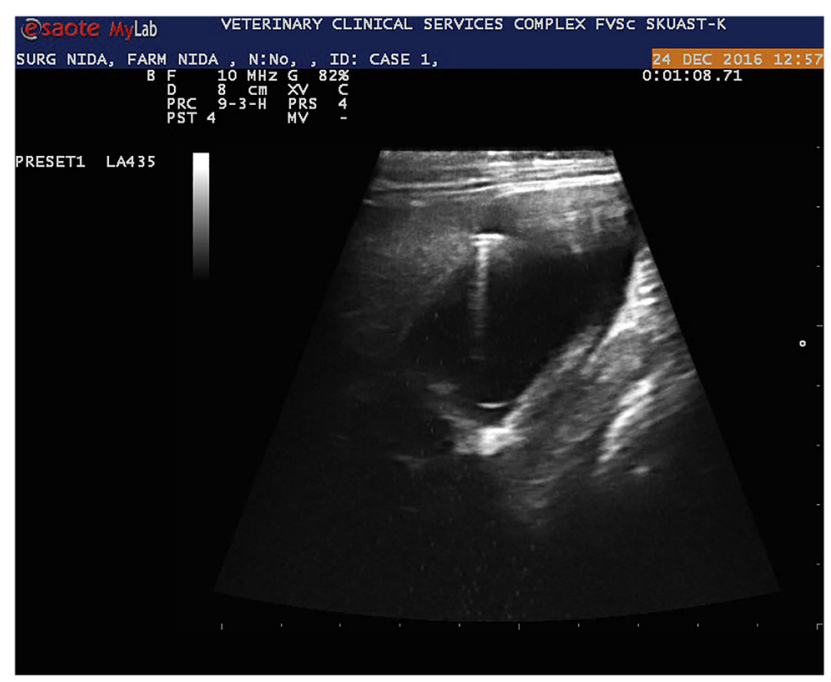

Fig. 2. Ultrasonographic longitudinal view of the hypodermic needle in an abdominal cyst

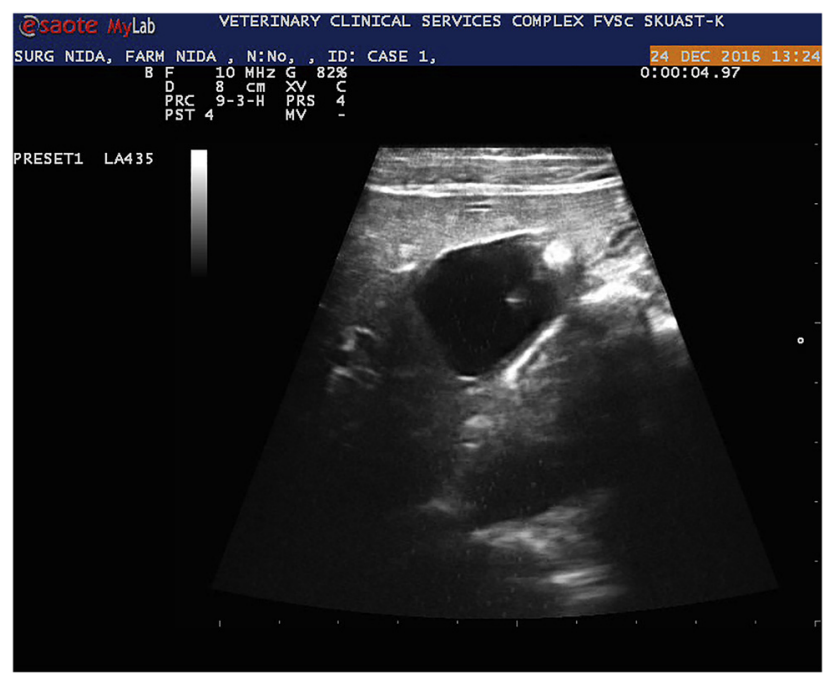

Fig. 3. Ultrasonographic cross-sectional view of the hypodermic needle in an abdominal cyst

The values of cyst volume and diameter reduction over time were analysed by Friedman's non-parametric and Wilcoxon's paired rank sum tests using SPSS Statistics 20.

\section{RESULTS}

All the animals included in this study were free of clinical signs. Their haematological and biochemical parameters were within the normal range throughout the study period.

The sclerotherapy procedure was accomplished without difficulty and safely in standing animals after local infiltration anaesthesia at the puncture site. Although two animals showed slight body movement, the needle did not get displaced and adverse consequences were not encountered.

Out of the six cysts in total (one per animal) located by USG, four were located in the abdominal cavity adjacent to the solid viscera and two were hepatic. All the cysts appeared globular or oval anechoic structures with smooth margins but compartmentalisation and/or debris was not visualised (corresponding to Gharbi type-I hydatid cysts in humans). The aspirated fluid from all the cysts also did not contain scolex or debris.

On USG, the minimum cyst volume recorded before PAIR was $2.0 \mathrm{~mL}$ and the maximum $11.0 \mathrm{~mL}$. Their diameter ranged from 1.06 to $4.21 \mathrm{~cm}$. The mean $\pm \mathrm{SD}$ diameter and volume was $2.67 \pm 1.30 \mathrm{~cm}$ and $7.11 \pm 3.40$ $\mathrm{mL}$, respectively.

The only goat included in the study showed moderate restlessness immediately after the procedure and was injected $4.0 \mathrm{mg}$ dexamethasone intravenously (IV) (Dexona, Sarabhai Zydus, India). Within a period of $10 \mathrm{~min}$, the distress signs subsided. The animal did not develop any complications thereafter.

On day 7, the cysts were located in five of the six animals included in the trial. The minimum volume and diameter of the cysts was $1.9 \mathrm{~mL}$ and $1.24 \mathrm{~cm}$, respectively. The maximum volume and diameter was $7.5 \mathrm{~mL}$ and $1.90 \mathrm{~cm}$, respectively. The sclerotherapy procedure was repeated in all these animals.

On day 30, the cysts were again detected in all the five animals. Their volume ranged from 1.5 to $6.3 \mathrm{~mL}$ and their diameter from 0.90 to $1.83 \mathrm{~cm}$. The decrease in the volume and diameter of the cysts continued subsequently. However, in one animal the cyst volume had increased from $3.0 \mathrm{~mL}$ on day 7 to $4.3 \mathrm{~mL}$ on day 30 . On day 90 , its volume was $4.0 \mathrm{~mL}$.

Data analysis by Friedman's test indicated highly significant $(P<0.001)$ volume and diameter reduction over time (Tables 1 and 2). Wilcoxon's signed rank test also revealed significant $(P<0.05)$ reduction in cyst volume and diameter between all the observation intervals except cyst volume between day 7 and day 30 (Tables 3 and 4).

Table 1. Diameter $(\mathrm{cm})$ of the abdominal cysts in sheep and goat subjected to PAIR (Friedman's non-parametric test)

\begin{tabular}{lclll}
\hline Ultrasonography days & Day 0 & Day 7 & Day 30 & Day 90 \\
\hline Minimum diameter & 1.06 & $0.00^{* * * *}$ & $0.00^{* * *}$ & $0.00^{* * * *}$ \\
Maximum diameter & 4.21 & $1.90^{* * *}$ & $1.83^{* * *}$ & $1.40^{* * *}$ \\
Mean diameter & 2.67 & $1.43^{* * *}$ & $1.26^{* * *}$ & $0.00^{* * *}$ \\
Standard deviation & 1.30 & 0.74 & 0.68 & 0.00 \\
\hline
\end{tabular}

PAIR $=$ percutaneous aspiration-injection-reaspiration.

Friedman's test $P$ value $<0.001$; ${ }^{* * *}$ Highly significant.

Table 2. Volume (mL) of the cysts in sheep and goat subjected to PAIR (Friedman's non-parametric test)

\begin{tabular}{lrrll}
\hline Ultrasonography days & Day 0 & Day 7 & Day 30 & Day 90 \\
\hline Minimum volume & 2.00 & $0.00^{* * *}$ & $0.00^{* * *}$ & $0.00^{* * *}$ \\
Maximum volume & 11.00 & $7.50^{* * *}$ & $6.30^{* * *}$ & $4.00^{* * *}$ \\
Mean volume & 7.11 & $3.67^{* * *}$ & $2.78^{* * *}$ & $0.00^{* * *}$ \\
Standard deviation & 3.40 & 2.72 & 2.24 & 0.00 \\
\hline
\end{tabular}

PAIR $=$ percutaneous aspiration-injection-reaspiration.

Friedman's test $P$ value $<0.001$; ${ }^{* * *}$ Highly significant. 
Table 3. Diameter $(\mathrm{cm})$ of the abdominal cysts in sheep and goat subjected to PAIR (Wilcoxon's paired rank sum test)

\begin{tabular}{lcccccccccccc}
\hline Interval (days) & \multicolumn{2}{c}{$0-7$} & \multicolumn{2}{c}{$0-30$} & \multicolumn{2}{c}{$0-90$} & \multicolumn{2}{c}{$7-30$} & \multicolumn{2}{c}{$7-90$} & $30-90$ \\
\hline Days & 0 & 7 & 0 & 30 & 0 & 90 & 7 & 30 & 7 & 90 & 30 & 90 \\
Minimum diameter & 1.06 & 0 & 1.06 & 0 & 1.06 & 0 & 0 & 0 & 0 & 0 & 0 & 0 \\
Maximum diameter & 4.21 & 1.90 & 4.21 & 1.83 & 4.21 & 0 & 1.90 & 1.83 & 1.90 & 0 & 1.83 & 0 \\
Mean & 2.67 & 1.43 & 2.67 & 1.26 & 2.67 & 0 & 1.43 & 1.26 & 1.43 & 0 & 1.26 & 0 \\
Standard deviation & 1.30 & 0.74 & 1.30 & 0.68 & 1.30 & 0 & 0.74 & 0.68 & 0.74 & 0 & 0.68 & 0 \\
Wilcoxon significance & \multicolumn{2}{c}{0.028} & \multicolumn{2}{c}{0.028} & \multicolumn{2}{c}{0.028} & \multicolumn{2}{c}{0.043} & 0.043 & 0.043 \\
\hline
\end{tabular}

PAIR $=$ percutaneous aspiration-injection-reaspiration.

Table 4. Volume (mL) of the abdominal cysts in sheep and goat subjected to PAIR (Wilcoxon's paired rank sum test)

\begin{tabular}{lcccccccccccc}
\hline Interval (days) & \multicolumn{2}{c}{$0-7$} & \multicolumn{2}{c}{$0-30$} & $0-90$ & & $7-30$ & \multicolumn{2}{c}{$7-90$} & $30-90$ \\
\hline Day & 0 & 7 & 0 & 30 & 0 & 90 & 7 & 30 & 7 & 90 & 30 & 90 \\
Minimum volume & 2.0 & 0 & 2.0 & 0 & 2.0 & 0 & 0 & 0 & 0 & 0 & 0 & 0 \\
Maximum volume & 11.0 & 7.5 & 11.0 & 6.3 & 11.0 & 0 & 7.5 & 6.3 & 7.5 & 0 & 6.3 & 0 \\
Mean & 7.11 & 3.67 & 7.11 & 2.78 & 7.11 & 0 & 3.67 & 2.78 & 3.67 & 0 & 2.78 & 0 \\
Standard deviation & 3.40 & 2.72 & 3.40 & 2.24 & 3.40 & 0 & 2.72 & 2.24 & 2.72 & 0 & 2.24 & 0 \\
Wilcoxon significance & \multicolumn{2}{c}{0.028} & \multicolumn{2}{c}{0.028} & 0.028 & & 0.345 & 0.043 & 0.042 \\
\hline
\end{tabular}

PAIR $=$ percutaneous aspiration-injection-reaspiration.

\section{DISCUSSION}

In Kashmir, a large number of stray dogs (up to 90,000 in Srinagar city alone) are always found roaming throughout the grazing lands, resulting in a high $(7.97 \%)$ prevalence of cystic echinococcosis in sheep (Rashid et al., 2017).

Only two of the six animals included in this trial had a cyst in the liver. Cysticercus tenuicollis is frequently found attached to the greater omentum, mesentery and visceral organs. The hydatid cysts develop in the lungs and the liver (Ahmad et al., 2018). Ultrasonographic features of the hydatid cysts in the liver of sheep are the presence of rounded, unilocular and anechoic structures. The borders of the cysts are mostly well defined. The interior of the cysts may contain echogenic particulate materials, septations, or fine echoes (Hussain and Elrashidy, 2014). In humans, E. granulosus cysts following primary infection are most commonly detected in the liver (65\%) and the lungs (25\%). During a secondary infection following cyst rupture, the scolices can grow within the peritoneum, pleura, bronchial tree and/or bile ducts and/or be carried to distant organs via the blood stream (Khuroo, 2002).

The maximum diameter of a cyst in the animals included in this study was $4.21 \mathrm{~cm}$. Cysts of larger diameter (6.0-10.0 $\mathrm{cm}$ ) have been noticed in sheep livers (Hussain and Elrashidy, 2014). In humans, the size of hepatic cysts ranges from 1.0 to $15.0 \mathrm{~cm}$, and cyst growth varies from 1.0 to 30.0 $\mathrm{mm}$ per year (Khuroo, 2002). In $63.88 \%$ of the human patients the abdominal cysts were found to be less than 10.0 $\mathrm{cm}$ in diameter (Dar et al., 2015).

The aspirated cystic fluid in all the small ruminants included in this study was colourless. Its biochemical analysis could not be accomplished. In humans the hepatic cyst fluid was found to be crystal clear, had a specific gravity of 1.012 , contained salts, enzymes, proteins and toxic substances, and was also antigenic (Khuroo, 2002).
All the animals included in this study received local anaesthetic infiltration to allow painless passage of the hypodermic needle in the abdominal wall. In human beings, the PAIR procedure has also been accomplished successfully under local infiltration of the puncture site (Rajesh et al., 2013).

Sedation was avoided in the animals included in this first trial involving ruminant animals to prevent recumbency, abdominal distension and a restricted field of abdominal USG visualisation. Although the procedure was accomplished smoothly in sheep but the goat showed distress. The procedure should therefore be preferably performed under sedation or general anaesthesia, particularly in temperamentally active goats.

A standard 18-G hypodermic needle was used satisfactorily to undertake PAIR in the animals included in this trial. Either hypodermic needles (18 G-22 G) or pigtail catheters (5-9 Fr. G) are used in human patients (Filice et al., 2000; Rajesh et al., 2013).

In our animals half to one-third of the aspirated fluid volume per cyst was replaced with hypertonic saline. As per the routine practice in humans, the volume of the scolicidal agent infused is equal to one-third of the aspirated volume (Lohani et al., 2013). Hypertonic saline (20\%) has satisfactorily been used in cystic hydatid disease of the liver in humans (Acunas et al., 1992). It has been recommended as a scolicidal agent by the World Health Organization (Rajesh et al., 2013). Saline sclerotherapy has the advantage of being comparatively less painful to the patient (Elgimez et al., 2007). Alcohol (95\%), Betadine (10\% povidone iodine $+1 \%$ free iodine), albendazole and mebendazole are also used for this purpose (Ustunsoz et al., 1999; Bosanac and Lisanin, 2000; Raymond et al., 2003; Rajesh et al., 2013).

In the present study, the hypertonic saline was retained in the cyst cavity for $10 \mathrm{~min}$ before aspiration. In human 
beings, the time allowed for the sclerotic agent to act generally varies from 5 to $30 \mathrm{~min}$ but may even be extended to $4 \mathrm{~h}$ (Larssen et al., 2003; Yang et al., 2006). In one study the scolicidal agent was not aspirated after injection into the cyst (Giorgio et al., 1992).

The single goat included in this trial showed restlessness and bleating immediately after the procedure but the distress signs subsided following the administration of dexamethasone. Cyst fluid spillage can occur during surgery in 2-25\% of the cases, resulting in anaphylaxis (Khuroo, 2002). Following the PAIR technique, such spillage was reported in a lower number $(0.52 \%)$ of patients (Golemanov et al., 2011). Long-term follow-up has shown that secondary echinococcosis does not occur following percutaneous drainage (Khuroo et al., 1997).

None of the more than 1,000 human patients treated with percutaneous puncture showed anaphylactic shock or peritoneal dissemination (Filice et al., 2000). Inadvertent liver puncture leading to intraabdominal bleeding or bile peritonitis, anaphylaxis, allergic reactions and biliary communications are the rarely encountered complications (Khuroo et al., 1997; Filice et al., 2000; Golemanov et al., 2011).

The second session of sclerotherapy was performed on day 7 in five out of the six animals. The cyst size was reduced subsequently and all cysts except one were not visible on USG examination by day 90 . The cyst in one animal persisted up to day 90. The treatment is considered successful if on USG the scar tissue or the normal tissue replaces the cyst area (Jusufovic and Zerem, 2011). Solidification and calcification have also been noticed in a few human patients followed up for one year. The reduction in hepatic cyst size is evaluated after three to six months (Rajesh et al., 2013). Multiple sessions of sclerotherapy performed at different time intervals yield better results and reduce recurrence (Belcher and Hull, 1969; Bean and Rodan, 1985; Sonnenberg et al., 1994).

The clinical, haematological and blood biochemical profile in animals included in this study were all within the normal range for most of the study period. Small, uncomplicated cysts cause no clinical signs and are seen incidentally while using diagnostic imaging techniques (Sarkar et al., 2016). Hussain and Elrashidy (2014) also reported the lack of significant differences in haematological parameters between healthy and cyst-bearing sheep. They found significant alterations in the biochemical parameters in sheep with hepatic cysts. In our trial the data pertaining to all the six animals (four with peritoneal and two with hepatic cysts) were pooled. This might have resulted in the nonsignificant changes in their biochemical values.

In conclusion, sclerotherapy using hypertonic saline (20\%) is a minimally invasive, inexpensive, effective and safe interventional USG technique for the treatment of abdominal cysts in sheep and goats. However, the procedure needs further evaluation after using different sclerotic agents of varying concentrations and different durations of their retention in the cysts in a sufficient number of animals with cysts.

\section{REFERENCES}

Acunas, B., Rozanes, I., Celik, L., Minareci, O., Acunas, G., Alper, A., Ariogul, O. and Gökmen, E. (1992): Purely cystic hydatid disease of the liver: treatment with percutaneous aspiration and injection of hypertonic saline. Radiology 182, 541-543.

Ahmad, P. A., Sharma, R., Katoch, R., Godara, R. and Yadav, A. (2018): Prevalence of larval tapeworm in sheep and goats of Jammu, India. Indian J. Small Rum. 24, 304-307.

Akhan, O., Dincer, A., Gököz, A., Sayek, I., Havlioglu, S., Abbasoglu, O., Eryilmaz, M., Besim, A. and Baris, I. (1993): Percutaneous treatment of abdominal hydatid cysts with hypertonic saline and alcohol. An experimental study in sheep. Invest. Radiol. 28, 121-127.

Aygun, E., Sahin, M., Odev, K., Vatansev, C., Aksoy, F. and Paksoy, Y. (2001): The management of liver hydatid cysts by percutaneous drainage. Can. J. Surg. 44, 203-209.

Bean, W. J. and Rodan, B. A. (1985): Hepatic cysts: treatment with alcohol. Am. J. Roentgenol. 144, 237-241.

Belcher, H. V. and Hull, H. C. (1969): Nonparasitic cysts of the liver: report of three cases. Surgery 65, 427-431.

Boland, L. E., Hardie, R. J., Gregory, S. P. and Lamb, C. R. (2003): Ultrasound-guided percutaneous drainage as the primary treatment for prostatic abscesses and cysts in dogs. J. Am. Anim. Hosp. Assoc. 39, 151-159.

Bosanac, Z. B. and Lisanin, L. (2000): Percutaneous drainage of hydatid cyst in the liver as a primary treatment: review of 52 consecutive cases with long-term follow-up. Clin. Radiol. 55, 839-848.

Cai, Z. H., Wang, W. M., Shi, J. R., Liang, Y. J., Ma, Y. J. and Zhang, J. Z. (2016): Comparison of ultrasound interventional and laparoscopic surgeries for ovarian cyst pediculotorsion. J. Acute Dis. 5, 458-461.

Dar, H. M., Wani, M., Ahmad, M. M. and Salam, P. (2015): A study on evaluation of biliary leaks following hydatid liver surgeries. Arch. Clinic. Exp. Surg. 4, 49-94.

Elgimez, H., Gok ,V., Oztoprak, I., Atalar, M., Cetin, A., Arslan, M., Gultekin, Y. and Solak, O. (2007): Comparison of CT-guided sclerotherapy with using $95 \%$ ethanol and $20 \%$ hypertonic saline for managing simple renal cyst. Korean J. Radiol. 8, 512519.

Filice, C., Brunetti, E., Bruno, R. and Crippa, F. G. (2000): Letters to the editor: percutaneous aspiration in the treatment of hydatid liver cysts. Gut 47, 156-157.

Giorgio, A., Tarantino, L., Francica, G., Mariniello, N., Aloisio, T., Soscia, E. and Pierri, G. (1992): Unilocular hydatid liver cysts: treatment with US-guided, double percutaneous aspiration and alcohol injection. Radiology 184, 705-710.

Golemanov, B., Grigorov, N., Mitova, R., Genov, J., Vuchev, D., Tamarozzi, F. and Brunetti, E. (2011): Efficacy and safety of PAIR for cystic echinococcosis: experience on a large series of patients from Bulgaria. Am. J. Trop. Med. Hyg. 84, 48-51.

Hussein, H. A. and Elrashidy, M. (2014): Ultrasonographic features of the liver with cystic echinococcosis in sheep. Vet. Rec. Open 1, e000004. https://doi.org/10.1136/vropen-2013000004. 
Jusufovic, R. and Zerem, E. (2011): Percutaneous treatment of symptomatic non-parasitic benign liver cysts with $20 \% \mathrm{NaCl}$ solution. Med. Arh. 65, 35-37.

Khuroo, M. S. (2002): Hydatid disease: current status and recent advances. Ann. Saudi Med. 22, 56-64.

Khuroo, M. S., Wani, N. A. and Javid, G. (1997): Percutaneous drainage compared with surgery for hepatic hydatid cysts. N. Engl. J. Med. 337, 881-887.

Khuroo, M. S., Zargar, S. A. and Mahajan, R. (1991): Echinococcus granulosus cysts in the liver: management with percutaneous drainage. Radiology 180, 141-145.

Larssen, T. B., Rosendahl, K., Horn, A., Jensen, D. K. and Rorvik, J. (2003): Single-session alcohol sclerotherapy in symptomatic benign hepatic cysts performed with a time of exposure to alcohol of 10 min: initial results. Eur. Radiol. 13, 2627-2632.

Lohani, B., Gurung, G. and Paudel, S. (2013): PAIR for hepatic hydatid cyst: a study in tertiary care hospital in Nepal. J. Inst. Med. Nepal 35, 2.

Nasseri, S., Abrishami, A. and Malekzadeh, R. (2006): Percutaneous needle aspiration, injection, and reaspiration with or without benzimidazole coverage for uncomplicated hepatic hydatid cysts. Cochrane Database Syst. Rev. 2, CD003623.

Rajesh, R., Dalip, D. S., Anupam, J. and Jaisiram, A. (2013): Effectiveness of puncture-aspiration-injection-reaspiration in the treatment of hepatic hydatid cysts. Iran J. Radiol. 10, 68-73.
Rashid, A., Darzi, M. M., Mir, M. S., Dar, L. M., Mir, A., Kashani, S. B., Maharana, B. R., Patbandha, T. K. and Allaie, I. M. (2017): Prevalence of ovine cystic echinococcosis in Kashmir Valley, North India. Vet. Parasitol. Reg. Stud. Reports 10, $85-89$.

Raymond, A., Smego., Bhatti, S., Khaliq, A. A. and Beg, M. A. (2003): Percutaneous aspiration-injection-reaspiration drainage plus albendazole or mebendazole for hepatic cystic echinococcosis: a meta-analysis. Clin. Infect. Dis. 37, 1073-1083.

Sarkar, M., Pathania, R., Jhobta, A., Thakur, B. R. and Chopra, R. (2016): Cystic pulmonary hydatidosis. Lung India 33, 179-191.

Sonnenberg, E., Wroblicka, J. T. and D'Agostino, H. B. (1994): Symptomatic hepatic cysts: percutaneous drainage and sclerosis. Radiology 190, 387-392.

Ustunsoz, B., Akhan, O., Kamiloglu, M. A., Somuncu, I., Ugurel, M. S. and Cetiner, S. (1999): Percutaneous treatment of hydatid cysts of the liver: long-term results. Am. J. Roentgenol. 172, 91-96.

Yang, C. F., Liang, H. L., Pan, H. B., Lin, Y. H., Mok, K. T. and Lo, G. H. (2006): Single-session prolonged alcohol-retention sclerotherapy for large hepatic cysts. Am. J. Roentgenol. 187, 940-943.

Yoder, B. M. and Wolf, J. S. Jr. (2004): Long-term outcome of laparoscopic decortication of peripheral and peripelvic renal and adrenal cysts. J. Urol. 171, 583-587. 